\title{
The Hamiltonian mass of asymptotically anti-de Sitter space-times
}

\author{
Piotr T. Chruściel* and Gabriel Nagy ${ }^{\dagger}$ \\ Département de Mathématiques, Faculté des Sciences, Parc de Grandmont, F-37200 Tours, France
}

\begin{abstract}
We give a Hamiltonian definition of mass for spacelike hypersurfaces in space-times with metrics which are asymptotic to the anti-de Sitter one, or to a class of generalizations thereof. We present the results of [1] which show that our definition provides a geometric invariant for a spacelike hypersurface $\mathscr{S}$ embedded in a space-time $(\mathscr{M}, g)$. Some further global invariants are also given.
\end{abstract}

Let $\mathscr{S}$ be an $n$-dimensional spacelike hypersurface in a $n+1$-dimensional Lorentzian space-time $(\mathscr{M}, g)$. Suppose that $\mathscr{M}$ contains an open set $\mathscr{U}$ which is covered by a finite number of coordinate charts $\left(t, r, v^{A}\right)$, with $r \in[R, \infty)$, and with $\left(v^{A}\right)$ - local coordinates on some compact $n-1$ dimensional manifold $M$, such that $\mathscr{S} \cap \mathscr{U}=\{t=0\}$. Assume that the metric $g$ approaches a background metric $b$ of the form

$$
\begin{array}{r}
b=-a^{-2}(r) d t^{2}+a^{2}(r) d r^{2}+r^{2} h, \\
h=h_{A B}\left(v^{C}\right) d v^{A} d v^{B},
\end{array}
$$

with $a(r)=1 / \sqrt{r^{2} / \ell^{2}+k}$, where $h$ is a Riemannian metric on $M, k$ is a constant, and $\ell$ is a strictly positive constant related to the cosmological constant $\Lambda$ by the formula $2 \Lambda=-n(n-1) / \ell^{2}$ (somewhat more general metrics are considered in [1]). For example, if $h$ is the standard round metric on $S^{2}$ and $k=1$, then $b$ is the anti-de Sitter metric. To make the approach rates precise it is convenient to introduce an orthonormal frame for $b$,

$$
e_{0}=a(r) \partial_{t}, \quad e_{1}=\frac{1}{a(r)} \partial_{r}, \quad e_{A}=\frac{1}{r} \beta_{A},
$$

with $\beta_{A}$ - an $h$-orthonormal frame on $(M, h)$, so that $b_{a b}=b\left(e_{a}, e_{b}\right)=\eta_{a b}$ - the usual Minkowski matrix $\operatorname{diag}(-1,+1, \cdots,+1)$. We then require that the frame components $g_{a b}$ of $g$ with respect to the frame (2) satisfy [29]

$$
e^{a b}=O\left(r^{-\beta}\right), \quad e_{a}\left(e^{b c}\right)=O\left(r^{-\beta}\right), \quad b_{a b} e^{a b}=O\left(r^{-\gamma}\right),
$$

where $e^{a b}=g^{a b}-b^{a b}$, with

$$
\beta>n / 2, \quad \gamma>n .
$$

(The $n+1$ dimensional Schwarzschild-anti de Sitter metrics (15) satisfy (3) with $\beta=n$, and with $\gamma=2 n$. The boundary conditions (3)-(汪), and the overall approach here stem from [2, Section 5].) We stress that we do not assume existence of global frames on the asymptotic region: when $M$ is not parallelizable, then conditions (3)-(4) should be understood as the requirement of existence of a covering of $M$ by a finite number of open sets $\mathscr{O}_{i}$ together with frames defined on $\left[R_{0}, \infty\right) \times \mathscr{O}_{i}$ satisfying (3)-(因). Suppose further, for simplicity, that $g$ satisfies the vacuum Einstein equations with a cosmological constant,

$$
R_{\mu \nu}-\frac{g^{\alpha \beta} R_{\alpha \beta}}{2} g_{\mu \nu}=-\Lambda g_{\mu \nu},
$$

similarly for $b$. (The existence of a large family of such $g$ 's follows from the work in [3, 4h.) A geometric Hamiltonian analysis [1, 5], based on the formalism of [6], leads to the following expression for the Hamiltonian associated to the flow of a vector field $X$, assumed to be a Killing vector field of the background $b: 30$ ]

$$
\begin{gathered}
m(\mathscr{S}, g, b, X)=\frac{1}{2} \int_{\partial \mathscr{S}} \mathbb{U}^{\alpha \beta} d S_{\alpha \beta}, \\
\mathbb{U}^{\nu \lambda}=\mathbb{U}_{\beta}^{\nu \lambda} X^{\beta}+\frac{1}{8 \pi}\left(\sqrt{\left|\operatorname{det} g_{\rho \sigma}\right|} g^{\alpha[\nu}-\sqrt{\left|\operatorname{det} b_{\rho \sigma}\right|} b^{\alpha[\nu}\right) \delta_{\beta}^{\lambda]} \stackrel{\circ}{\nabla}_{\alpha} X^{\beta}, \\
\mathbb{U}_{\beta}^{\nu \lambda}=\frac{2\left|\operatorname{det} b_{\mu \nu}\right|}{16 \pi \sqrt{\left|\operatorname{det} g_{\rho \sigma}\right|}} g_{\beta \gamma} \stackrel{\circ}{\nabla}_{\kappa}\left(e^{2} g^{\gamma[\nu} g^{\lambda] \kappa}\right) \\
e=\sqrt{\left|\operatorname{det} g_{\rho \sigma}\right|} / \sqrt{\left|\operatorname{det} b_{\mu \nu}\right|} .
\end{gathered}
$$

\footnotetext{
* Supported in part by the Polish Research Council grant KBN 2 P03B 073 15. E-mail: chrusciel@univ-tours.fr

$\dagger$ Supported by a grant from Région Centre. E-mail: nagy@gargan.math.univ-tours.fr
} 
It is appropriate to make a few comments here: In [5] the starting point of the analysis is the Hilbert Lagrangian for vacuum Einstein gravity, $\mathcal{L}=\sqrt{-\operatorname{det} g_{\mu \nu}} g^{\alpha \beta} R_{\alpha \beta} / 16 \pi$. With our signature $(-+\cdots+)$ one needs to repeat the analysis in [5] with $\mathcal{L}$ replaced by $\sqrt{-\operatorname{det} g_{\mu \nu}}\left(g^{\alpha \beta} R_{\alpha \beta}-2 \Lambda\right) / 16 \pi$, and without making the assumption $n+1=4$ done there. The final expression for the Hamiltonian (6) ends up to coincide with that obtained when $\Lambda=0$ and $n+1=4$. Equation (7) coincides with that derived in [0] except for the term $-\sqrt{\left|\operatorname{det} b_{\rho \sigma}\right|} b^{\alpha[\nu} \delta_{\beta}^{\lambda]} \nabla_{\alpha}^{\circ} X^{\beta}$. This term does not depend on the metric $g$, and such terms can be freely added to the Hamiltonian because they do not affect the variational formula that defines a Hamiltonian. From an energy point of view such an addition corresponds to a choice of the zero point of the energy. We note that in our context $m(\mathscr{S}, g, b, X)$ would not converge if the term $-\sqrt{\left|\operatorname{det} b_{\rho \sigma}\right|} b^{\alpha[\nu} \delta_{\beta}^{\lambda]} \stackrel{\circ}{\nabla}_{\alpha} X^{\beta}$ were not present in (7). The extension to non-vacuum models is straightforward and leads to the same Hamiltonian for several standard matter models; the simplest way to prove this is by following the arguments in [7].

We show in [1] that the vacuum Einstein equations together with the decay conditions (3)-(4) and the asymptotic behavior of the frame components of the $b$-Killing vector fields,

$$
X^{a}=O(r), \stackrel{\circ}{\nabla}_{a} X^{b}=O(r),
$$

guarantee that the integrals (6) are convergent. Further, it is easy to see that under the asymptotic conditions (3)-(估) the volume integrals appearing in the variational formulae in [5] are convergent, the undesirable boundary integrals in the variational formulae of [5] vanish, so that the integrals (6) do indeed provide Hamiltonians on the space of fields satisfying (3)-(伍). (Compare [8 for an alternative, essentially equivalent, Hamiltonian approach.) This singles out the charges (5) amongst various alternative expressions because Hamiltonians are uniquely defined, up to the addition of a constant, on each path connected component of the phase space. The key advantage of the Hamiltonian approach is precisely this uniqueness property, which does not seem to have a counterpart in the Noether charge analysis [9] (cf., however [10, 11]), or in Hamilton-Jacobi type arguments [12].

To define the integrals (6) we have fixed a model background metric $b$, as well as an orthonormal frame as in (2); this last equation requires the corresponding coordinate system $\left(t, r, v^{A}\right)$ as in (1). Hence, the background structure required in our analysis consists of a background metric and a background coordinate system. While this is reasonably satisfactory from a Hamiltonian point of view, in which each choice of background structure defines an associated phase space, there is an essential potential geometric ambiguity in the integrals (6) that arises as follows: let $g$ be any metric such that its frame components $g^{a b}$ tend to $\eta^{a b}$ as $r$ tends to infinity, in such a way that the integrals $m(\mathscr{S}, g, b, X)$ given by (6) (labelled by all the background Killing vector fields $X$ or perhaps by a subset thereof) converge. Consider another coordinate system $\left(\hat{t}, \hat{r}, \hat{v}^{A}\right)$ with the associated background metric $\hat{b}$ :

$$
\hat{b}=-a^{-2}(\hat{r}) d \hat{t}^{2}+a^{2}(\hat{r}) d \hat{r}^{2}+\hat{r}^{2} \hat{h}, \quad \hat{h}=h_{A B}\left(\hat{v}^{C}\right) d \hat{v}^{A} d \hat{v}^{B},
$$

together with an associated frame $\hat{e}^{a}$,

$$
\hat{e}_{0}=a(\hat{r}) \partial_{\hat{t}}, \quad \hat{e}_{1}=\frac{1}{a(\hat{r})} \partial_{\hat{r}}, \quad \hat{e}_{A}=\frac{1}{\hat{r}} \hat{\beta}_{A},
$$

and suppose that in the new hatted coordinates the integrals defining the charges $m(\hat{\mathscr{S}}, g, \hat{b}, \hat{X})$ converge again. An obvious way of obtaining such coordinate systems is to make a coordinate transformation

$$
t \rightarrow \hat{t}=t+\delta t, r \rightarrow \hat{r}=r+\delta r, v^{A} \rightarrow \hat{v}^{A}=v^{A}+\delta v^{A},
$$

with $\left(\delta t, \delta r, \delta v^{A}\right)$ decaying sufficiently fast:

$$
\begin{gathered}
\hat{t}=t+O\left(r^{-1-\beta}\right), \quad e_{a}(\hat{t})=\ell \delta_{a}^{0}+O\left(r^{-1-\beta}\right), \\
\hat{r}=r+O\left(r^{1-\beta}\right), \quad e_{a}(\hat{r})=\frac{\delta_{a}^{1}}{\ell}+O\left(r^{1-\beta}\right), \\
\hat{v}^{A}=v^{A}+O\left(r^{-1-\beta}\right), \quad e_{a}\left(\hat{v}^{A}\right)=\delta_{a}^{A}+O\left(r^{-1-\beta}\right),
\end{gathered}
$$

and with analogous conditions on second derivatives; this guarantees that the hatted analogue of Equations (3) and (4) will also hold. In [1] we prove that under coordinate transformations (12) the integrals (6) remain unchanged:

$$
m(\mathscr{S}, g, b, X)=m(\hat{\mathscr{S}}, g, \hat{b}, \hat{X}) \text {. }
$$

Here, if $X=X^{\mu}\left(t, r, v^{A}\right) \partial_{\mu}$, then the vector field $\hat{X}$ is defined using the same functions $X^{\mu}$ of the hatted variables. The proof is a computation of the change of the integrand of (6) under the change of coordinates (11); one notices 
that all terms obtained by linearizing in $\left(\delta t, \delta r, \delta v^{A}\right)$ can be written as a total divergence, and therefore give no contribution; this is reminiscent of a divergence identity which is used for a similar calculation for asymptotically flat space-times. One finally checks that under the boundary conditions (3)-(㺼) the correction terms give vanishing contribution in the limit $r \rightarrow \infty$.

One does not expect all the requirements in (3)-(4) to be necessary: for metrics which are asymptotically flat at $i_{0}$ it is sufficient to impose conditions on the induced metric and the extrinsic curvature of the hypersurface $\mathscr{S}$ to obtain a well defined mass, and one expects the same to be the case here. However, the decay rates imposed are sharp in the following sense: consider the metric $g=\hat{b}$, with the hatted coordinates defined as

$$
\hat{r}=r+\frac{\zeta}{r^{n / 2-1}}, \quad \hat{v}^{A}=v^{A},
$$

where $\zeta$ is a constant. Thus, if $\hat{b}$ is the anti-de Sitter metric, then $g$ is again anti-de Sitter metric in a different coordinate system, which does not differ too much from the original one; in particular the metric approaches asymptotically the standard version of the anti-de Sitter metric in the unhatted coordinates. Then the components of $g$ with respect to the ON frame associated to the unhatted background $b$ satisfy (3) with $\beta=\gamma=n / 2$; a calculation shows that $g$ has non-vanishing mass integral (6) with respect to the unhatted background $b$. The same coordinate transformation leads to a non-zero Abbott-Deser 13 mass of $g=\hat{b}$ with respect to $b$.

It should be stressed that we do not know a priori that the hatted coordinates are related to the unhatted ones by the simple coordinate transformation (11) with $\left(\delta t, \delta r, \delta v^{A}\right)$ decaying as $r \rightarrow \infty$, or behaving in some controlled way - the behavior of $\left(\delta t, \delta r, \delta v^{A}\right)$ could in principle be very wild. The main technical result of [1] is the proof that this is not the case: under the hypothesis that $(M, h)$ is a sphere with a round metric, or that $(M, h)$ has non-positive Ricci tensor and constant Ricci scalar[31] we show that all coordinate transformations which leave $\mathscr{S}$ invariant (so that $\hat{t}=t$ ) and that preserve the decay conditions (3)-(4) are compositions of a map satisfying (19) with an isometry of the background. In order to obtain a geometric invariant of $\mathscr{S}$ it remains thus to study the behavior of the integrals (6) under isometries of $b$ preserving $\mathscr{S}$. If $\Phi$ is such an isometry, the fact that $\mathbb{U}^{\alpha \beta}$ is a tensor density immediately yields the formula

$$
m\left(\mathscr{S}, \Phi^{*} g, b,\left(\Phi_{*}\right)^{-1} X\right)=m(\mathscr{S}, g, b, X) .
$$

(Here $\Phi^{*}$ is the pull-back, and $\Phi_{*}$ is the push-forward map. When understood in a passive manner, $\Phi^{*} g$ is simply the metric $g$ expressed in the new coordinates, while $\left(\Phi_{*}\right)^{-1} X$ is the vector field $X$ expressed in the new coordinates.) This has the effect that the integrals (6) are reshuffled amongst each other under isometries of $b$, in a way determined by the representation of the group of isometries of $b$ on the space of Killing vectors, and leads to the following:

1. Let $M$ be the $n-1$ dimensional sphere ${ }^{n-1} S$ with a round metric $h$, normalized so that the substitution $k=1$ in (11) leads to a metric $b$ which is the $n+1$ dimensional anti-de Sitter metric. The space $\mathscr{K}_{\mathscr{S} \perp}$ of $b$-Killing vector fields normal to $\mathscr{S} \cap \mathscr{U}$ is spanned by vector fields $K_{(\mu)}$ which on $\mathscr{S}$ take the form $K_{(\mu)}=N_{(\mu)} e_{0}, \mu=0, \cdots, n$, where $N_{(0)}=\sqrt{\frac{r^{2}}{\ell^{2}}+1}, N_{(i)}=\frac{x^{i}}{\ell}$, and $x^{i}=r n^{i}, r$ being the coordinate which appears in (1), while $n^{i} \in{ }^{n-1} S \subset \mathbb{R}^{n}$. The group Iso $(\mathscr{S}, b)$ of isometries $\Phi$ of $b$ which map $\mathscr{S}$ into $\mathscr{S}$ coincides with the homogeneous Lorentz group $O(1, n)$; it acts on $\mathscr{K}_{\mathscr{S} \perp}$ by push-forward. It can be shown that for each such $\Phi$ there exists a Lorentz transformation $\Lambda=\left(\Lambda_{(\beta)}^{(\alpha)}\right): \mathbb{R}^{n+1} \rightarrow \mathbb{R}^{n+1}$ so that for every $X=X^{(\alpha)} K_{(\alpha)} \in \mathscr{K}_{\mathscr{S} \perp}$ we have

$$
\left(\Phi_{*} X\right)^{(\alpha)}=\Lambda_{(\beta)}^{(\alpha)} X^{(\beta)} .
$$

We set

$$
m_{(\mu)} \equiv m\left(\mathscr{S}, g, b, K_{(\mu)}\right)
$$

it follows that the number

$$
m^{2}(\mathscr{S}, g)=\left|\eta^{(\mu)(\nu)} m_{(\mu)} m_{(\nu)}\right|,
$$

where $\eta^{(\mu)(\nu)}=\operatorname{diag}(-1,+1, \cdots,+1)$ is the Minkowski metric on $\mathbb{R}^{n+1}$, is an invariant of the action of $I s o(\mathscr{S}, b)$. Further, if we define $m(\mathscr{S}, g)$ to be positive if $m^{(\mu)}:=\eta^{(\mu)(\nu)} m_{(\nu)}$ is spacelike, and we take the sign of $m(\mathscr{S}, g)$ to coincide with that of $m^{(0)}$ if $m^{(\mu)}$ is timelike or null, then $m(\mathscr{S}, g)$ is invariant under the action of the group Iso ${ }^{\uparrow}(\mathscr{S}, b)$ of time-orientation preserving Lorentz transformations. The number $m(\{t=0\}, g)$ so defined coincides with the mass 
parameter $m$ of the Kottler ("Schwarzschild - anti-de Sitter") metrics in dimension $n+1=4$, and it is proportional to the parameter $m$ which occurs in the $(n+1)$-dimensional generalizations of the Kottler metrics 14

$$
g=-\left(1-\frac{2 m}{r^{n-2}}+\frac{r^{2}}{\ell^{2}}\right) d t^{2}+\left(1-\frac{2 m}{r^{n-2}}+\frac{r^{2}}{\ell^{2}}\right)^{-1} d r^{2}+r^{2} h
$$

with $h$ - a round metric of scalar curvature $(n-1)(n-2)$ on a $(n-1)$-dimensional sphere $(c f .$, e.g., [15]).

Consider the remaining Killing vector fields $L_{(\alpha)(\beta)}$ of $b$ :

$$
L_{(\alpha)(\beta)}=\eta_{(\alpha)(\sigma)} y^{(\sigma)} \frac{\partial}{\partial y^{(\beta)}}-\eta_{(\beta)(\sigma)} y^{(\sigma)} \frac{\partial}{\partial y^{(\alpha)}} .
$$

Here the coordinates $y^{(\alpha)}$ are the coordinates on $\mathbb{R}^{n+1}$ obtained by isometrically embedding — into $\mathbb{R}^{n+1}$ - the $\{t=0\}$ section of $n+1$-dimensional anti-de Sitter space-time equipped with the Minkowski metric $\eta^{(\mu)(\nu)}$. It can be checked that under the action of $\operatorname{Iso}(\mathscr{S}, b)$ the integrals

$$
Q_{(\mu)(\nu)} \equiv m\left(\mathscr{S}, g, b, L_{(\mu)(\nu)}\right)
$$

transform as the components of a two-covariant antisymmetric tensor. One then obtains a geometric invariant of $\mathscr{S}$ by calculating

$$
Q \equiv Q_{(\mu)(\nu)} Q_{(\alpha)(\beta)} \eta^{(\mu)(\alpha)} \eta^{(\nu)(\beta)}
$$

In dimension $3+1$ another independent global geometric invariant is obtained from

$$
Q^{*} \equiv Q_{(\mu)(\nu)} Q_{(\alpha)(\beta)} \epsilon^{(\mu)(\alpha)(\nu)(\beta)}
$$

In higher dimensions further invariants are obtained by calculating $\operatorname{tr}\left(P^{2 k}\right), 2 \leq 2 k \leq(n+1)$, where $P^{(\alpha)}(\beta)=$ $\eta^{(\alpha)(\mu)} Q_{(\mu)(\beta)}$. (In this notation $Q$ given by Equation (17) equals $\operatorname{tr}\left(P^{2}\right)$.)

2. Let $M$ be a compact $n-1$ dimensional manifold with a metric $h$ of constant scalar curvature and with nonpositive Ricci tensor, and let $b$ take the form (1), with $a(r)=1 / \sqrt{r^{2} / \ell^{2}+k}$, and with $k=0$ or -1 according to whether the Ricci scalar of $h$ vanishes or not. We show in $[\mathbb{1}$ that for such metrics the space of $b$-Killing vector fields normal to $\mathscr{S}$ consists of vector fields of the form $X(\lambda)=\lambda \partial_{t}, \lambda \in \mathbb{R}$, and that

$$
m(\mathscr{S}, g) \equiv m(\mathscr{S}, g, b, X(1))
$$

is background independent, hence a geometric invariant 32. Some other geometric invariants can be obtained from the integrals (6) when Killing vectors which are not necessarily normal to $\mathscr{S}$ exist, using invariants of the action of the isometry group of $b$ on the space of Killing vectors. If the Ricci tensor of $M$ is strictly negative no other Killing vectors exist. On the other hand, if $h$ is a flat torus, then each $h$-Killing vector provides a global geometric invariant via the integrals (6).

The number $m(\mathscr{S}, g)$ defined in each case above is our proposal for the geometric definition of total mass of $\mathscr{S}$ in $(\mathscr{M}, g)$. We note that its multiplicative normalization in $n+1=4$ dimensions is determined by the requirement of the correct Newtonian limit when $M=S^{2}$ and $\Lambda=0$, while the additive one is determined by imposing that the background models have vanishing energy. In higher dimensions it appears appropriate to keep the same multiplicative factor for the Lagrangian $\mathcal{L}$, whenever a Kaluza-Klein reduction applies.

The results described above can be reformulated in a purely Riemannian context 16].

It is natural to study the invariance of the mass when $\mathscr{S}$ is allowed to move in $\mathscr{M}$. A complete answer would require establishing an equivalent of our analysis of admissible coordinate transformations in a space-time setting. The difficulties that arise in the corresponding problem for asymptotically Minkowskian metrics 17 suggest that this might be a considerably more delicate problem, which we plan to analyse in the future. It should be stressed that this problem mixes two different issues, one being the potential background dependence of ( 6 ), another one being the possibility of energy flowing in or out through the timelike conformal boundary of space-time.

It should be pointed out that there exist several alternative methods of defining mass in asymptotically anti-de Sitter space-time — using coordinate systems [18, 19], preferred foliations [20], generalized Komar integrals [21, conformal techniques [22, 23, 24, or ad-hoc methods [13]; an extended discussion can be found in [2], Section 5]. Each of those approaches suffers from some potential ambiguities, so that the question of the geometric character of the definition of mass given there arises as well. Let us briefly describe the relationship of the results presented 
here to some of those works. Consider, first, the Abbott-Deser approach [13], which seems to have been most often used. A precise comparison is difficult to carry out because in Ref. [13] the boundary conditions which should be assumed are not spelled out in detail. As already pointed out, the coordinate transformation (13) gives a non-zero Abbott-Deser mass to the anti-de Sitter metric, so the boundary conditions do matter. Assuming that the authors of 13] had in mind boundary conditions which are at least as restrictive as our conditions (3)-(何), a straightforward but tedious calculation shows that the Abbott-Deser mass coincides with the Hamiltonian mass advocated here. One needs then to face the same ambiguities as we do, and our results in [1] can be interpreted as proving the existence of a geometric invariant which can be calculated using Abbott-Deser type integrals. It should be stressed, however, that while the Hamiltonian approach is universal and leads to unique - up to a constant - functionals on each connected component of phase space, no results about either uniqueness or universality of the approach of [13] are known to us.

Consider, next, the Hamiltonian approach of [8]; here the question of equivalence of the different Hamiltonian formalisms used in [5] and in [8] arises. Those formalisms are identical in spirit — both are Hamiltonian — but differ in various details. We note that the boundary conditions (3)-(何) are weaker than the conditions imposed in [8]. It can be checked that under (3)-(4) the formalism of [8] is a special case of the geometric Hamiltonian formalism of [5], when applied to asymptotically anti-de Sitter space-times, so the Hamiltonian part of our analysis in [1] can be thought of as an extension of the results of [8] to larger phase spaces arising naturally in this context. An advantage of the geometric Hamiltonian formalism of Kijowski and Tulczyjew is that it allows the use of both a manifestly four-dimensional covariant formalism, and of a $3+1 \mathrm{ADM}$ one. The potential ambiguities in a geometric definition of mass that occur in [8] are identical to the ones described above.

As another example, we note the potential ambiguity in the mass defined by the conformal methods in [22, 23 , related to the possibility of existence of smooth conformal completions which are not smoothly conformally equivalent. In those works one assumes existence of smooth completions, while our conditions would - roughly speaking correspond to $C^{1, \alpha}$ conformal completions, $\alpha>1 / 2$, hence our conditions (3)-(4) are satisfied in the setup of [22, 23] (compare [2] for a detailed discussion in the static case). It can be shown (A. Ashtekar, private communication) that the mass of 22, 23] coincides with the Abbott-Deser one; what has been said above implies that - under the asymptotic conditions of 22, 23] - it also coincides with the Hamiltonian mass described here. The results proved in [1] can be used to show that no inequivalent conformal completions of the kind considered in [22, 23] exist, establishing the invariant character of the framework of [22, 23.

Let us, finally, turn our attention to the AdS/CFT motivated definitions of mass (cf., e.g., 22, 24, 25, 26] and references therein). It seems that there might be a belief in the string community that, at least in odd spacetime dimensions, there is no systematic way to start from the Einstein-Hilbert action and arrive unambiguously at conserved quantities. Our results in [1] show that such a belief is incorrect, in the space of metrics asymptotic to the backgrounds considered here, as well as for those which asymptote to the more general backgrounds considered in [1. It should be pointed out, however, that the Hamiltonian approach gives Hamiltonians which are only defined up to a constant, which leaves ample room for non-zero "Casimir energies", the occurrence of which might well be justified by other physical considerations. On the other hand, in a Hamiltonian approach the only natural choice of the additive constant seems to be zero. In any case, it is not clear whether or not the AdS/CFT based approaches lead to geometric invariants of hypersurfaces in space-times, in the sense presented here.

We note that a similar problem for the ADM mass of asymptotically flat initial data sets has been settled in [27, 28] (see also [17]). Our treatment in [1] is a non-trivial extension to the current setup of the methods of [28].

Acknowledgements: PTC wishes to thank M. Herzlich, S. Ilias, A. Polombo and A.El Soufi for useful discussions. Suggestions from an anonymous referee for improvements of the manuscript are acknowledged.

[1] P. Chruściel and G. Nagy (2001), gr-qc/0110014.

[2] P. Chruściel and W. Simon, Jour. Math. Phys. 42, 1779 (2001), gr-qc/0004032.

[3] H. Friedrich, Jour. Geom. and Phys. 17, 125 (1995).

[4] J. Kánnár, Class. Quantum Grav. 13, 3075 (1996).

[5] P. Chruściel, Ann. Inst. H. Poincaré 42, 267 (1985).

[6] J. Kijowski and W. Tulczyjew, A Symplectic Framework for Field Theories, vol. 107 of Lecture Notes in Physics (Springer, New York, Heidelberg, Berlin, 1979).

[7] J. Kijowski, Gen. Rel. Grav. 9, 857 (1978).

[8] M. Henneaux and C. Teitelboim, Commun. Math. Phys. 98, 391 (1985).

[9] A. Trautman, in Gravitation: an introduction to current research, edited by L. Witten (Wiley, 1962).

[10] R. Wald and A. Zoupas, Phys. Rev. D61, 084027 (16 pp.) (2000), gr-qc/9911095. 
[11] B. Julia and S. Silva, Class. Quantum Grav. 17, 4733 (2000), gr-qc/0005127.

[12] J. Brown and J. York, Jr., Phys. Rev. D47, 1407 (1993).

[13] L. Abbott and S. Deser, Nucl. Phys. B195, 76 (1982).

[14] G. Horowitz and R. Myers, Phys. Rev. D59, 026005 (12 pp.) (1999), hep-th/9808079.

[15] C. Cadeau and E. Woolgar, Class. Quantum Grav. pp. 527-542 (2001), gr-qc/0011029.

[16] P. Chruściel and M. Herzlich, math.DG/0110035.

[17] P. Chruściel, Commun. Math. Phys. 120, 233 (1988).

[18] W. Boucher, G. Gibbons, and G. Horowitz, Phys. Rev. D 30, 2447 (1984).

[19] G. Gibbons, S. Hawking, G. Horowitz, and M. Perry, Commun. Math. Phys. 88, 295 (1983).

[20] G. Gibbons, Class. Quantum Grav. 16, 1677 (1999).

[21] A. Magnon, Jour. Math. Phys. 26, 3112 (1985).

[22] A. Ashtekar and S. Das, Class. Quantum Grav. 17, L17 (2000), hep-th/9911230.

[23] A. Ashtekar and A. Magnon, Class. Quantum Grav. 1, L39 (1984).

[24] V. Balasubramanian and P. Kraus, Commun. Math. Phys. 208, 413 (1999), hep-th/9902121.

[25] R. Emparan, C. Johnson, and R. Myers, Phys. Rev. D60, 104001 (14 p.) (1999), hep-th/9903238.

[26] R. Myers, Phys. Rev. D60, 046002 (12 p.) (1999), hep-th/9903203.

[27] R. Bartnik, Comm. Pure Appl. Math. 39, 661 (1986).

[28] P. Chruściel, in Topological Properties and Global Structure of Space-Time, edited by P. Bergmann and V. de Sabbata (Plenum Press, New York, 1986), pp. 49-59, URL http://www.phys.univ-tours.fr/ piotr/scans.

[29] The summation convention is used throughout. We use Greek indices for coordinate components and lower-case Latin indices for the tetrad ones; upper-case Latin indices run from 2 to $n$ and are associated either to coordinates or to frames on $M$, in a way which should be obvious from the context. Finally Greek bracketed indices $(\alpha)$ etc. refer to objects defined outside of space-time, such as the Killing algebra $\mathscr{K}_{\mathscr{S} \perp}$, or an exterior embedding space, and are also summed over.

[30] The integral over $\partial \mathscr{S}$ should be understood by a limiting process, as the limit as $R$ tends to infinity of integrals over the sets $t=0, r=R . d S_{\alpha \beta}$ is defined as $\left.\left.\frac{\partial}{\partial x^{\alpha}}\right\rfloor \frac{\partial}{\partial x^{\beta}}\right\rfloor d x^{0} \wedge \cdots \wedge d x^{n}$, with $\rfloor$ denoting contraction; $g$ stands for the space-time metric unless explicitly indicated otherwise. Square brackets denote antisymmetrization with an appropriate numerical factor ( $1 / 2$ for two indices), and $\nabla^{\circ}$ denotes covariant differentiation with respect to the background metric $b$.

[31] Related results under less restrictive conditions can be found in [1].

[32] If the scalar curvature of $h$ vanishes, then Einstein equations require the constant $k$ in the metric to vanish. In that case there arises an ambiguity in the definition of mass related to the possibility of rescaling $t$ and $r$ without changing the form of the metric, which rescales the mass. (This freedom does not occur when $k$ is non-zero.) This ambiguity can be removed by arbitrarily choosing some normalization for the $h$-volume of $M$, e.g. $4 \pi$ in dimension $n+1=4$. 